OPEN ACCESS

Edited by:

Olivier Feron

Université catholique de Louvain,

Belgium

Reviewed by:

Yongguang Tao,

Central South University, China

Sung Eun Kim,

Korea University, South Korea

${ }^{*}$ Correspondence:

Jiao Liu

2018683073@gzhmu.edu.cn

Daolin Tang

daolin.tang@utsouthwestern.edu

Specialty section:

This article was submitted to Pharmacology of Anti-Cancer Drugs, a section of the journal

Frontiers in Pharmacology

Received: 10 September 2021

Accepted: 29 November 2021

Published: 10 December 2021

Citation:

Liu J, Kang $R$ and Tang D (2021) The

Art of War: Ferroptosis and

Pancreatic Cancer.

Front. Pharmacol. 12:773909.

doi: 10.3389/fphar.2021.773909

\section{The Art of War: Ferroptosis and Pancreatic Cancer}

\author{
Jiao Liu ${ }^{1 *}$, Rui Kang ${ }^{2}$ and Daolin Tang $^{2 *}$ \\ ${ }^{1}$ The Third Affiliated Hospital of Guangzhou Medical University, Guangzhou, China, ${ }^{2}$ Department of Surgery, UT Southwestern \\ Medical Center, Dallas, TX, United States
}

Pancreatic cancer is a devastating gastrointestinal cancer, characterized by late diagnosis, low treatment success rate, and poor survival prognosis. The most common pathological type of pancreatic cancer is pancreatic ductal adenocarcinoma (PDAC), which is mainly driven by the K-Ras oncogene. Ferroptosis was originally described as Ras-dependent cell death, but is now defined as lipid peroxidation-mediated regulated necrosis, accompanied by excessive activation of the autophagy degradation pathway and limited membrane repair capacity. The impaired ferroptotic pathway is involved in many types of cancer, including PDAC. On the one hand, the chronic inflammation caused by ferroptotic damage contributes to the formation of K-Ras-driven PDAC. On the other hand, drug-induced ferroptosis is an emerging strategy to suppress tumor growth in established PDAC. In this mini-review, we outline the core process of ferroptosis, discuss the regulatory mechanism of ferroptosis in PDAC, and highlight some of the challenges of targeting ferroptosis in PDAC therapy.

Keywords: autophagy, ferroptosis, pancreatic cancer, tumorigenesis, targeted therapy

\section{INTRODUCTION}

Pancreatic ductal adenocarcinoma (PDAC) is the most common pathological type of pancreatic cancer, accounting for more than $90 \%$ of all pancreatic malignancies (Kleeff et al., 2016). The KRAS gene is mutated in approximately $85-90 \%$ of PDAC and is the main driver of pancreatic tumorigenesis (Buscail et al., 2020). Despite improvements in surgical techniques, chemotherapy regimens, and the introduction of neoadjuvant chemoradiotherapy or chemoimmunotherapy, PDAC still accounts for 3\% of all cancers and $7 \%$ of all cancer deaths in the United States (Siegel et al., 2021). Due to modifiable lifestyle factors, such as high-fat diets, the incidence of PDAC is increasing (Heinen et al., 2009). The American Cancer Society estimates that by 2021, there will be 60,430 pancreatic cancer diagnoses and 48,220 deaths in the United States (Siegel et al., 2021). From 2014 to 2021, the general 5-years survival rate of patients with PDAC slowly increased from 6 to $10 \%$. The poor outcomes of PDAC are mainly due to the late diagnosis of the disease and its resistance to treatments involving cell death. Thus, it is essential to understand the cell death machinery of PDAC and to develop new treatment strategies (Chen et al., 2021a). Recent studies have shown that inducing ferroptotic cell death may be an attractive therapy for various types of cancer, including PDAC (Su et al., 2020; Chen et al., 2021b; Shi et al., 2021).

\section{THE CORE MECHANISM OF FERROPTOSIS}

The term "ferroptosis" was first proposed to describe a type of iron-dependent non-apoptotic cell death in cancer cells with RAS mutations (Dixon et al., 2012). Today, the core molecular mechanism 


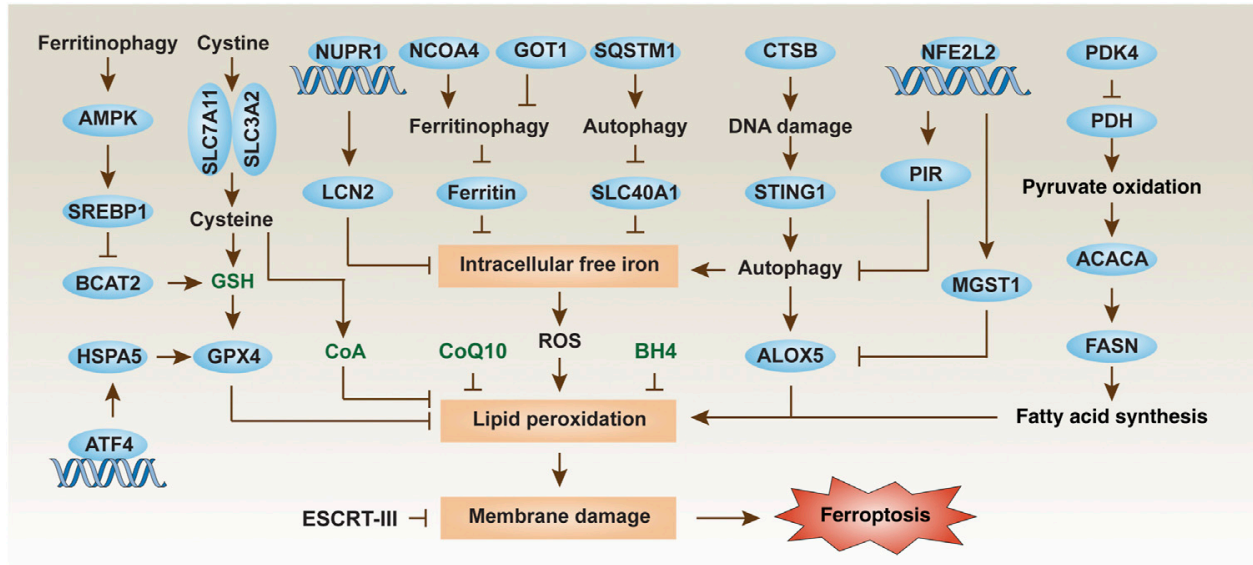

FIGURE 1 | Regulation mechanisms and signaling pathways of ferroptosis in PDAC cells. Ferroptosis is an iron-dependent cell death driven by lipid peroxidation and subsequent membrane damage. The level of ferroptosis in PDAC cells can be regulated in multiple ways, including through autophagic degradation, transcription factors, and metabolic pathways.

of ferroptosis is involved in the production of lipid peroxidation and subsequent plasma membrane damage (Stockwell et al., 2017; Tang et al., 2020a). During ferroptosis, reactive oxygen species (ROS) can be obtained from the iron-dependent Fenton reaction, a mitochondrial electron transport chain-mediated reaction, or a membrane NADPH oxidase (NOX)-mediated reaction (Xie et al., 2016). However, the connection between the multiple sources of ROS production during ferroptosis remains obscure. Three antioxidant systems [glutathione (GSH), coenzyme Q10 (CoQ10), and tetrahydrobiopterin (BH4)] have been shown to inhibit ferroptosis caused by oxidative damage (Dixon et al., 2012; Bersuker et al., 2019a; Kraft et al., 2020; Dai et al., 2020a). Among them, the system $\mathrm{xc}^{-}$-GSH-glutathione peroxidase 4 (GPX4) axis plays a major role in blocking lipid peroxidation during ferroptosis (Figure 1). System $\mathrm{xc}^{-}$, a transmembrane protein complex composed of two subunits, namely solute carrier family 7 member 11 (SLC7A11) and solute carrier family 3 member 2 (SLC3A2), mediates the entry of cystine into cells to exchange glutamate. Once cystine enters cells, it is quickly reduced to cysteine, which is required for GSH synthesis. GSH is a substrate for the antioxidant GPX4 to prevent the accumulation of toxic lipids. GPX4 and SLC7A11 also regulate other types of non-ferroptotic death, indicating that it may not be possible to distinguish them based on a single molecular event (Ran et al., 2006; Canli et al., 2016; Kang et al., 2018; Chen et al., 2021c). Alternatively, apoptosis inducing factor mitochondria associated 2 (AIFM2) plays a GPX4-independent role in limiting ferroptosis by sustaining the production of reduced GSH (Bersuker et al., 2019b; Doll et al., 2019) or increasing membrane repair (Dai et al., 2020a).

Polyunsaturated fatty acids (PUFAs) are the main peroxidation substrates for ferroptosis in cell membranes. Consequently, increasing PUFA synthesis can increase the sensitivity to ferroptosis, which is positively regulated by acylcoenzyme A (CoA) synthetase long-chain family member 4 (ACSL4) (Dixon et al., 2015; Yuan et al., 2016; Doll et al.,
2017; Kagan et al., 2017). Apart from this, the biosynthesis of plasmalogens from peroxisomes also contributes to ferroptosis (Zou et al., 2020a). PUFAs are found in most foods, but are highest in fatty fish, seeds, and nuts. It is possible to adjust the sensitivity to ferroptosis by changing the content and type of dietary fat. Finally, two families of lipid peroxidases [lipoxygenase (ALOX) and cytochrome P450 oxidoreductase (POR)] play a context-dependent role in mediating toxic lipid production during ferroptosis (Yang et al., 2016; Wenzel et al., 2017; Chu et al., 2019; Li et al., 2020; Zou et al., 2020b; Yan et al., 2021). However, the molecular effectors of ferroptosis have not yet been identified. As a conservative membrane repair mechanism, the calcium-dependent endosomal sorting complexes required for transport (ESCRT)-III pathway can be activated to separate damaged membranes in various cancer cells (including PDAC) during ferroptosis (Dai et al., 2020b).

\section{FERROPTOSIS IN PANCREATIC TUMORIGENESIS}

The inflammatory process has become a key mediator of the development and progression of pancreatic cancer. Consistent with this notion, ferroptotic damage can release damageassociated molecular pattern molecules (DAMPs), thereby creating an inflammatory tumor microenvironment for tumor growth and development (Bianchi, 2007). For example, the conditional depletion of Gpx4 in the pancreas or a high-iron diet accelerates the development of $\mathrm{Kras}^{G 12 D}$-driven pancreatic tumors in mice (Dai et al., 2020c). This process is mediated by the release of nuclear DAMP 8-hydroxydeoxyguanosine (8-OH-dG) by ferroptotic cells. The released $8-\mathrm{OH}-\mathrm{dG}$ activates the stimulator of interferon response CGAMP interactor 1 (STING1, also known as TMEM173) pathway in surrounding macrophages, thereby inducing the release of cytokines (e.g., interleukin 6) to maintain the chronic inflammatory 
TABLE 1 | Main regulators of ferroptosis in PDAC.

\begin{tabular}{|c|c|c|c|}
\hline Name & Function & Mechanism & Refs. \\
\hline NCOA4 & Promoter of ferroptosis & Induce autophagic degradation of ferritin & Hou et al. (2016) \\
\hline SQSTM1 & Promoter of ferroptosis & Induce autophagic degradation of SLC4OA1 & Li et al. (2021a) \\
\hline STING1 & Promoter of ferroptosis & Induce autophagy-dependent ferroptosis & Li et al. (2020) \\
\hline ALOX5 & Promoter of ferroptosis & Induce lipid ROS production & Li et al. (2020) \\
\hline CTSB & Promoter of ferroptosis & Induce DNA damage and lysosomal dysfunction & Kuang et al. (2020), Nagakannan et al. (2021) \\
\hline ACACA & Promoter of ferroptosis & Increase fatty acid synthesis & Song et al. (2021) \\
\hline FASN & Promoter of ferroptosis & Increase fatty acid synthesis & Song et al. (2021) \\
\hline $\mathrm{PDH}$ & Promoter of ferroptosis & Increase pyruvate oxidation & Song et al. (2021) \\
\hline AMPK & Promoter of ferroptosis & Inhibit BACT2 expression & Wang et al. (2020) \\
\hline SREBP1 & Promoter of ferroptosis & Inhibit BACT2 expression & Wang et al. (2020) \\
\hline SLC7A11 & Repressor of ferroptosis & Increase GSH or CoA synthesis & Badgley et al. (2020), Zhang et al. (2019), Badgley et al. (2020) \\
\hline GOT1 & Repressor of ferroptosis & Inhibit autophagic degradation of ferritin & Kremer et al. (2021) \\
\hline SLC40A1 & Repressor of ferroptosis & Promote iron export & Li et al. (2021a) \\
\hline Ferritin & Repressor of ferroptosis & Promote iron storage & Hou et al. (2016) \\
\hline ATF4 & Repressor of ferroptosis & Induce HSPA5 expression & Zhu et al. (2017) \\
\hline HSPA5 & Repressor of ferroptosis & Inhibit GPX4 degradation & Zhu et al. (2017) \\
\hline GPX4 & Repressor of ferroptosis & Inhibit lipid ROS production & Zhu et al. (2017), Liu et al. (2021a) \\
\hline PDK4 & Repressor of ferroptosis & Inhibit pyruvate oxidation & Song et al. (2021) \\
\hline BCAT2 & Repressor of ferroptosis & Increase GSH synthesis & Wang et al. (2020) \\
\hline MTOR & Repressor of ferroptosis & Inhibit autophagy-dependent ferroptosis & Liu et al. (2021a) \\
\hline NFE2L2 & Repressor of ferroptosis & Inhibit expression of antioxidant gene & Kuang et al. (2021), Hu et al. (2021) \\
\hline MGST1 & Repressor of ferroptosis & Inhibit oxidative stress & Kuang et al. (2021) \\
\hline PIR & Repressor of ferroptosis & Inhibit oxidative DNA damage & Kuang et al. (2021) \\
\hline POLG & Repressor of ferroptosis & Inhibit mitochondrial DNA damage-dependent autophagy & Li et al. (2020) \\
\hline TFAM & Repressor of ferroptosis & Inhibit mitochondrial DNA damage-dependent autophagy & Li et al. (2020) \\
\hline NUPR1 & Repressor of ferroptosis & Increase LCN2 expression & Liu et al. (2021b) \\
\hline LCN2 & Repressor of ferroptosis & Promote iron export & Liu et al. (2021b) \\
\hline ESCRT-III & Repressor of ferroptosis & Inhibit membrane repair & Dai et al. (2020b) \\
\hline
\end{tabular}

microenvironment of pancreatic tumorigenesis driven by Kras $^{G 12 D}$ (Dai et al., 2020c). These results explain the basic aspects of the inflammatory tumor microenvironment mediated by ferroptotic death in PDAC. Ferroptotic PDAC cells can also release KRAS ${ }^{\mathrm{G} 12 \mathrm{D}}$ protein into the extracellular space, and macrophages take up KRAS ${ }^{\mathrm{G} 12 \mathrm{D}}$ protein through advanced glycosylation end-product specific receptor (AGER, best known as RAGE), which eventually leads to macrophage polarization for tumor growth (Dai et al., 2020d). In contrast, the conditional deletion of Slc7a11 in the pancreas inhibits $\mathrm{Kras}^{\mathrm{G} 12 \mathrm{D}}$ / $T p 53^{R 172 H}$ mutation-driven pancreatic tumors in mice (Badgley et al., 2020), suggesting that additional Tp53 mutations may transform the carcinogenic effects of ferroptotic damage into anticancer effects in Kras ${ }^{G 12 D}$-driven PDAC. In general, these animal studies show that ferroptosis plays a dual role in pancreatic tumorigenesis, depending on gene deletions and mutations. It remains questionable whether genomic instability can produce genetic diversity in driving ferroptosis. It also needs to examine whether Gpx4 depletion has a similar effect in promoting mutant KRAS-driven tumorigenesis in other cancers, such as colorectal cancer and non-small cell lung cancer.

\section{REGULATION OF FERROPTOSIS IN PDAC}

The regulator of PDAC is involved in multiple molecules (Table 1). We discussed them from the following three perspectives, although this classification is rough considering the observed diversity of molecular mechanisms of ferroptosis.

\section{DEGRADATION SYSTEMS}

Macroautophagy (hereafter autophagy) and the ubiquitinproteasome system are the two degradation systems responsible for regulating cellular homeostasis ( $\mathrm{Li}$ et al., 2021b). Depending on the substrate being degraded, autophagic pathways play a significant role in pancreatic ferroptosis. A significant recent advance is that the autophagic degradation of the iron storage protein ferritin (a process also called ferritinophagy) (Hou et al., 2016) or the iron transporter solute carrier family 40 member 1 (SLC40A1, also known as ferroportin-1) (Li et al., 2021a) can increase the accumulation of free iron in cells, thereby inducing the Fenton reaction to produce ROS for ferroptosis in PDAC cells (Figure 1). More recently, glutamic-oxaloacetic transaminase 1 (GOT1) inhibition promotes ferroptosis in PDAC by inducing ferritinophagy to initiate iron-dependent oxidative damage (Kremer et al., 2021). Nuclear receptor coactivator 4 (NCOA4) (Hou et al., 2016) and sequestosome 1 (SQSTM1) (Li et al., 2021a) function as autophagy receptors to recognize and degrade ferritin or SLC40A1, respectively, during ferroptosis. However, identifying specific autophagy cargo receptors for ferroptosis remains a challenge. 
In addition to the classic ferroptosis activators (erastin and RSL3), zalcitabine (a drug used to treat human immunodeficiency virus infection) can cause mitochondrial damage, thereby activating STING1-dependent autophagy pathway and inducing ALOX5-related ferroptotic death in human PDAC cells (Li et al., 2020). The activation and release of cystatin B (CSTB, a lysosomal cysteine protease) can partially act as a mediator of ferroptosis by amplifying the STING1 pathway in human PDAC cells, arguing that ferroptosis is a form of autophagy-dependent lysosomal cell death coupled with a DNA sensor pathway (Kuang et al., 2020; Nagakannan et al., 2021).

In addition to autophagy, other degradation pathways also regulate ferroptosis by affecting the stability of GPX 4 protein. For example, transcription factor 4 (ATF4)-mediated heat shock protein family A (Hsp70) member 5 (HSPA5) expression related to endoplasmic reticulum stress can prevent the degradation of GPX4, thereby increasing the ferroptosis resistance of PDAC cells (Zhu et al., 2017). In contrast, highdose rapamycin can induce ferroptosis by promoting the degradation of GPX4 (Liu et al., 2021a). Because autophagy is generally used as a pro-survival pathway in PDAC, the induction of autophagy-dependent ferroptosis may provide a way to kill established PDAC cells (Görgülü et al., 2020). Nevertheless, clinically available autophagy inhibitors (e.g., chloroquine) may weaken the anticancer activity of ferroptosis activators ( $\mathrm{Li}$ et al., 2021a).

\section{METABOLIC PATHWAYS}

In the 1920s, Otto Warburg discovered that even in the presence of oxygen (aerobic glycolysis), cultured tumor cells had a high rate of glucose uptake and glycolysis (Vander Heiden et al., 2009). This Warburg effect triggers metabolic abnormalities, thereby promoting tumor growth or causing treatment resistance to transitional drugs (e.g., gemcitabine). Indeed, hyperglycemia occurs frequently in most patients with pancreatic cancer and is associated with a poor prognosis. Unexpectedly, ferroptosis in PDAC cells induced by system $\mathrm{xc}^{-}$inhibitors (erastin and sulfasalazine), but not GPX4 inhibitors (RSL3 and FIN56), requires high-glucose conditions (Song et al., 2021). In contrast, high-glucose limits staurosporine-induced cell death (Song et al., 2021). These results imply that glucose selectively confers susceptibility to ferroptosis, rather than apoptosis. In line with this notion, diabetes induced by a high-fat diet in mice also increased the anti-PDAC activity of ferroptosis inducers (Song et al., 2021).

Subsequent studies of metabolic mechanisms showed that pyruvate oxidation, but not pyruvate reduction, in mitochondria promotes ferroptosis in PDAC cells by activating acetyl-CoA carboxylase alpha (ACACA) and fatty acid synthase (FASN)-mediated fatty acid synthesis and subsequent ALOX5-dependent lipid peroxidation (Figure 1) (Song et al., 2021). This pro-ferroptosis process caused by glucose is negatively regulated by pyruvate dehydrogenase kinase 4 (PDK4), a repressor of pyruvate oxidation in mitochondria by blocking pyruvate dehydrogenase (PDH) (Song et al., 2021). An integrated metabolic reprogramming pathway may drive the production of fatty acid for ferroptosis.

In addition to glucose and lipids, amino acids also affect ferroptosis. For example, branched chain amino acid aminotransferase 2 (BCAT2) can inhibit ferroptosis in PDAC cells that is induced by system $\mathrm{xc}^{-}$inhibitors (erastin, sorafenib, and sulfasalazine) by producing sulfur amino acid for GSH synthesis (Wang et al., 2020). Moreover, system $\mathrm{xc}^{-}$-mediated cystine input is beneficial to the biosynthesis of $\mathrm{CoA}$, which plays a GSH-independent role in preventing IKE-induced ferroptosis in PDAC cells (Badgley et al., 2020). These findings provide a feedback mechanism for controlling ferroptosis through amino acid metabolism. Although the role of AMP-activated protein kinase (AMPK)-sterol regulatory element binding transcription factor 1 (SREBP1) pathway in ferroptosis is related to the type of cancer, the activation of this signaling pathway by ferritinophagy limits the expression of BCAT2 in PDAC cells (Wang et al., 2020). Since AMPK is an important kinase in various metabolic pathways, targeting the AMPK pathway combined with ferroptosis induction may be a strategy worthy of further exploration (Song et al., 2018; Lee et al., 2020). Recently, the endogenous metabolite itaconate induces iron death in PDAC cells by activating ferritinophagy (Qu et al., 2021), highlighting the new metabolic pathway of ferroptosis.

\section{STRESS SENSORS}

Another important research direction is to identify and study redox sensors in ferroptosis. Nuclear factor, erythroid 2-like 2 (NFE2L2, best known as NRF2) is a transcription factor that is sensitive to the redox state of cells under various cell death stimuli. NFE2L2 is negatively regulated by Kelch-like ECHassociated protein 1 (KEAP1), which targets NFE2L2 for protein degradation by the ubiquitin-proteasome system. In response to the stimulation of ferroptosis, autophagy receptor SQSTM1 binds and inhibits KEAP1, thereby promoting the activation of NFE2L2 and increasing the expression of antioxidant genes (Anandhan et al., 2020). Specifically, the NFE2L2-targeted genes microsomal glutathione S-transferase 1 (MGST1) (Kuang et al., 2021) and pirin (PIR) (Hu et al., 2021) have recently been identified as redox-sensitive repressors of ferroptosis in PDAC cells by binding ALOX5 or limiting the oxidative damage of DNA-mediated autophagy, respectively (Figure 1). These studies provide new insight into the complex mechanisms of NFE2L2-dependent redox signaling in PDAC. A key unanswered question is whether certain types of cell death are particularly associated with dysregulated NFE2L2 signaling.

In addition to NFE2L2, other stress-related transcription factors are also involved in the defense of ferroptosis. Nuclear protein 1, transcriptional regulator (NUPR1) is upregulated in the pancreas during various stresses, including ferroptotic damage (Liu et al., 2021b). As a pro-survival response, NUPR1-mediated expression of the iron exporter lipocalin 2 (LCN2) can prevent iron accumulation, thereby limiting oxidative damage and 
ferroptosis in PDAC cells (Figure 1) (Liu et al., 2021b). In a xenograft mouse model, the NUPR1 inhibitor ZZW-115 enhances the tumor suppressor effect of the ferroptosis activator IKE (Liu et al., 2021b). Together, targeting antioxidant transcription factors can enhance ferroptosismediated therapy in PDAC, although downstream effectors may be diverse.

\section{OPPORTUNITIES AND CHALLENGES}

The past few years have witnessed a rapid explosion of studies on ferroptosis and various cancers, including PDAC. Because PDAC responds weakly to all current treatment regimens, targeting the ferroptotic pathway may provide an alternative approach for this lethal disease. Exciting preclinical studies have shown that several drugs [artesunate (Eling et al., 2015) and zalcitabine (Li et al., 2020)] can suppress PDAC by inducing ferroptosis, although they may have off-target effects. Several therapy regimens related to ferroptosis (e.g., gemcitabine + sulfasalazine, sorafenib + sulfasalazine) have also been explored in PDAC in animal models. The ultimate goal of research is to develop clinically available drugs for the modulation of the ferroptosis pathway that can kill PDAC alone or in combination with other drugs. The challenge in developing effective drugs that induce ferroptosis is not to cause unnecessary side effects and to target specific sites in ferroptotic pathway. Under treatment selection pressure, resistance to treatment may appear due to the expansion of pre-existing subclonal populations or the evolution of drugresistant cells (Yang et al., 2014; Hangauer et al., 2017; Viswanathan et al., 2017). Although there are currently no clinical trials of ferroptosis-dependent treatment strategies, it is

\section{REFERENCES}

Anandhan, A., Dodson, M., Schmidlin, C. J., Liu, P., and Zhang, D. D. (2020). Breakdown of an Ironclad Defense System: The Critical Role of NRF2 in Mediating Ferroptosis. Cell Chem Biol 27, 436-447. doi:10.1016/ j.chembiol.2020.03.011

Badgley, M. A., Kremer, D. M., Maurer, H. C., DelGiorno, K. E., Lee, H. J., Purohit, V., et al. (2020). Cysteine Depletion Induces Pancreatic Tumor Ferroptosis in Mice. Science 368, 85-89. doi:10.1126/science.aaw9872

Bersuker, K., Hendricks, J. M., Li, Z., Magtanong, L., Ford, B., Tang, P. H., et al. (2019). The CoQ Oxidoreductase FSP1 Acts Parallel to GPX4 to Inhibit Ferroptosis. Nature 575, 688-692. doi:10.1038/s41586-019-1705-2

Bersuker, K., Hendricks, J. M., Li, Z., Magtanong, L., Ford, B., Tang, P. H., et al. (2019). The CoQ Oxidoreductase FSP1 Acts Parallel to GPX4 to Inhibit Ferroptosis. Nature 575, 688-692. doi:10.1038/s41586-019-1705-2

Bianchi, M. E. (2007). DAMPs, PAMPs and Alarmins: All We Need to Know about Danger. J. Leukoc. Biol. 81, 1-5. doi:10.1189/jlb.0306164

Buscail, L., Bournet, B., and Cordelier, P. (2020). Role of Oncogenic KRAS in the Diagnosis, Prognosis and Treatment of Pancreatic Cancer. Nat. Rev. Gastroenterol. Hepatol. 17, 153-168. doi:10.1038/s41575-019-0245-4

Canli, Ö., Alankuş, Y. B., Grootjans, S., Vegi, N., Hültner, L., Hoppe, P. S., et al. (2016). Glutathione Peroxidase 4 Prevents Necroptosis in Mouse Erythroid Precursors. Blood 127, 139-148. doi:10.1182/blood-2015-06-654194

Chen, X., Comish, P. B., Tang, D., and Kang, R. (2021). Characteristics and Biomarkers of Ferroptosis. Front Cel Dev Biol 9, 637162. doi:10.3389/ fcell.2021.637162 still necessary to establish the relationship between molecular characteristics and response to specific drugs in preclinical studies.

In addition to understanding the processes and functions of ferroptosis in the context of the ever-evolving complex cell death network, we need to develop powerful ferroptotic biomarkers in humans (Chen et al., 2021d). Since inflammation is a doubleedged sword, overcoming the immune side effects of ferroptotic damage may require a deeper understanding of the interaction between tumor cell death and immune cells. Several studies in other cancers have shown that DAMP released during erastininduced ferroptosis may activate adaptive tumor immunity to inhibit tumor growth (Efimova et al., 2020; Tang et al., 2020b). However, the occurrence of ferroptosis in dendritic cells or CD8 ${ }^{+}$ $\mathrm{T}$ cells can impair their anti-tumor function (Han et al., 2021; Ma et al., 2021). In the next few years, the impact of genetic mutations, degradation pathways, and metabolic plasticity on susceptibility to ferroptosis in the different component cells of the tumor microenvironment will be an active area of research (Chen et al., 2021e; Chen et al., 2021f; Tang et al., 2021).

\section{AUTHOR CONTRIBUTIONS}

All authors listed have made a substantial, direct, and intellectual contribution to the work, and approved it for publication.

\section{ACKNOWLEDGMENTS}

We thank Dave Primm (Department of Surgery, University of Texas Southwestern Medical Center) for his critical reading of the manuscript.

Chen, X., Kang, R., Kroemer, G., and Tang, D. (2021). Broadening Horizons: the Role of Ferroptosis in Cancer. Nat. Rev. Clin. Oncol. 18, 280. doi:10.1038/ s41571-020-00462-0

Chen, X., Kang, R., Kroemer, G., and Tang, D. (2021). Ferroptosis in Infection, Inflammation, and Immunity. J. Exp. Med. 218, 218. doi:10.1084/jem.20210518

Chen, X., Kang, R., Kroemer, G., and Tang, D. (2021f). Targeting Ferroptosis in Pancreatic Cancer: A Double-Edged Sword. Trends Cancer. 7, 891-901. doi:10.1016/j.trecan.2021.04.005

Chen, X., Li, J., Kang, R., Klionsky, D. J., and Tang, D. (2021e). Ferroptosis: Machinery and Regulation. Autophagy 17, 2054-2081. doi:10.1080/ 15548627.2020.1810918

Chen, X., Zeh, H. J., Kang, R., Kroemer, G., and Tang, D. (2021). Cell Death in Pancreatic Cancer: from Pathogenesis to Therapy. Nat. Rev. Gastroenterol. Hepatol. 18, 804-823. doi:10.1038/s41575-021-00486-6

Chu, B., Kon, N., Chen, D., Li, T., Liu, T., Jiang, L., et al. (2019). ALOX12 Is Required for P53-Mediated Tumour Suppression through a Distinct Ferroptosis Pathway. Nat. Cel Biol 21, 579-591. doi:10.1038/s41556-0190305-6

Dai, E., Han, L., Liu, J., Xie, Y., Kroemer, G., Klionsky, D. J., et al. (2020). Autophagy-dependent Ferroptosis Drives Tumor-Associated Macrophage Polarization via Release and Uptake of Oncogenic KRAS Protein. Autophagy 16, 2069-2083. doi:10.1080/15548627.2020.1714209

Dai, E., Han, L., Liu, J., Xie, Y., Zeh, H. J., Kang, R., et al. (2020). Ferroptotic Damage Promotes Pancreatic Tumorigenesis through a TMEM173/STINGdependent DNA Sensor Pathway. Nat. Commun. 11, 6339. doi:10.1038/s41467020-20154-8 
Dai, E., Meng, L., Kang, R., Wang, X., and Tang, D. (2020). ESCRT-III-dependent Membrane Repair Blocks Ferroptosis. Biochem. Biophys. Res. Commun. 522, 415-421. doi:10.1016/j.bbrc.2019.11.110

Dai, E., Zhang, W., Cong, D., Kang, R., Wang, J., and Tang, D. (2020). AIFM2 Blocks Ferroptosis Independent of Ubiquinol Metabolism. Biochem. Biophys. Res. Commun. 523, 966. doi:10.1016/j.bbrc.2020.01.066

Dixon, S. J., Lemberg, K. M., Lamprecht, M. R., Skouta, R., Zaitsev, E. M., Gleason, C. E., et al. (2012). Ferroptosis: an Iron-dependent Form of Nonapoptotic Cell Death. Cell 149, 1060-1072. doi:10.1016/j.cell.2012.03.042

Dixon, S. J., Winter, G. E., Musavi, L. S., Lee, E. D., Snijder, B., Rebsamen, M., et al. (2015). Human Haploid Cell Genetics Reveals Roles for Lipid Metabolism Genes in Nonapoptotic Cell Death. ACS Chem. Biol. 10, 1604-1609. doi:10.1021/acschembio.5b00245

Doll, S., Freitas, F. P., Shah, R., Aldrovandi, M., da Silva, M. C., Ingold, I., et al. (2019). FSP1 Is a Glutathione-independent Ferroptosis Suppressor. Nature 575, 693-698. doi:10.1038/s41586-019-1707-0

Doll, S., Proneth, B., Tyurina, Y. Y., Panzilius, E., Kobayashi, S., Ingold, I., et al. (2017). ACSL4 Dictates Ferroptosis Sensitivity by Shaping Cellular Lipid Composition. Nat. Chem. Biol. 13, 91-98. doi:10.1038/nchembio.2239

Efimova, I., Catanzaro, E., Van der Meeren, L., Turubanova, V. D., Hammad, H., Mishchenko, T. A., et al. (2020). Vaccination with Early Ferroptotic Cancer Cells Induces Efficient Antitumor Immunity. J. Immunother. Cancer 8, 8. doi:10.1136/jitc-2020-001369

Eling, N., Reuter, L., Hazin, J., Hamacher-Brady, A., and Brady, N. R. (2015). Identification of Artesunate as a Specific Activator of Ferroptosis in Pancreatic Cancer Cells. Oncoscience 2, 517-532. doi:10.18632/ oncoscience. 160

Görgülü, K., Diakopoulos, K. N., Kaya-Aksoy, E., Ciecielski, K. J., Ai, J., Lesina, M., et al. (2020). The Role of Autophagy in Pancreatic Cancer: From Bench to the Dark Bedside. Cells 99, 1063. doi:10.3390/cells9041063

Han, L., Bai, L., Qu, C., Dai, E., Liu, J., Kang, R., et al. (2021). PPARGmediated Ferroptosis in Dendritic Cells Limits Antitumor Immunity. Biochem. Biophys. Res. Commun. 576, 33-39. doi:10.1016/ j.bbrc.2021.08.082

Hangauer, M. J., Viswanathan, V. S., Ryan, M. J., Bole, D., Eaton, J. K., Matov, A., et al. (2017). Drug-tolerant Persister Cancer Cells Are Vulnerable to GPX4 Inhibition. Nature 551, 247-250. doi:10.1038/nature24297

Heinen, M. M., Verhage, B. A., Goldbohm, R. A., and van den Brandt, P. A. (2009). Meat and Fat Intake and Pancreatic Cancer Risk in the Netherlands Cohort Study. Int. J. Cancer 125, 1118-1126. doi:10.1002/ijc.24387

Hou, W., Xie, Y., Song, X., Sun, X., Lotze, M. T., Zeh, H. J., 3rd, et al. (2016). Autophagy Promotes Ferroptosis by Degradation of Ferritin. Autophagy 12, 1425-1428. doi:10.1080/15548627.2016.1187366

Hu, N., Bai, L., Dai, E., Han, L., Kang, R., Li, H., et al. (2021). Pirin Is a Nuclear Redox-Sensitive Modulator of Autophagy-dependent Ferroptosis. Biochem. Biophys. Res. Commun. 536, 100-106. doi:10.1016/j.bbrc.2020.12.066

Kagan, V. E., Mao, G., Qu, F., Angeli, J. P., Doll, S., Croix, C. S., et al. (2017). Oxidized Arachidonic and Adrenic PEs Navigate Cells to Ferroptosis. Nat. Chem. Biol. 13, 81-90. doi:10.1038/nchembio.2238

Kang, R., Zeng, L., Zhu, S., Xie, Y., Liu, J., Wen, Q., et al. (2018). Lipid Peroxidation Drives Gasdermin D-Mediated Pyroptosis in Lethal Polymicrobial Sepsis. Cell Host Microbe 24, 97-e4. doi:10.1016/j.chom.2018.05.009

Kleeff, J., Korc, M., Apte, M., La Vecchia, C., Johnson, C. D., Biankin, A. V., et al. (2016). Pancreatic Cancer. Nat. Rev. Dis. Primers 2, 16022. doi:10.1038/ nrdp.2016.22

Kraft, V. A. N., Bezjian, C. T., Pfeiffer, S., Ringelstetter, L., Müller, C., Zandkarimi, F., et al. (2020). GTP Cyclohydrolase $1 /$ Tetrahydrobiopterin Counteract Ferroptosis through Lipid Remodeling. ACS Cent. Sci. 6, 41-53. doi:10.1021/ acscentsci.9b01063

Kremer, D. M., Nelson, B. S., Lin, L., Yarosz, E. L., Halbrook, C. J., Kerk, S. A., et al. (2021). GOT1 Inhibition Promotes Pancreatic Cancer Cell Death by Ferroptosis. Nat. Commun. 12, 4860. doi:10.1038/s41467-021-24859-2

Kuang, F., Liu, J., Li, C., Kang, R., and Tang, D. (2020). Cathepsin B Is a Mediator of Organelle-specific Initiation of Ferroptosis. Biochem. Biophys. Res. Commun. 533, 1464-1469. doi:10.1016/j.bbrc.2020.10.035

Kuang, F., Liu, J., Xie, Y., Tang, D., and Kang, R. (2021). MGST1 Is a RedoxSensitive Repressor of Ferroptosis in Pancreatic Cancer Cells. Cel Chem Biol 28, 765. doi:10.1016/j.chembiol.2021.01.006
Lee, H., Zandkarimi, F., Zhang, Y., Meena, J. K., Kim, J., Zhuang, L., et al. (2020). Energy-stress-mediated AMPK Activation Inhibits Ferroptosis. Nat. Cel Biol 22, 225-234. doi:10.1038/s41556-020-0461-8

Li, C., Zhang, Y., Liu, J., Kang, R., Klionsky, D. J., and Tang, D. (2020). Mitochondrial DNA Stress Triggers Autophagy-dependent Ferroptotic Death. Autophagy 17, 948-960. doi:10.1080/15548627.2020.1739447

Li, C., Chen, X., Kang, R., Zeh, H., Klionsky, D. J., and Tang, D. (2021b). Regulation and Function of Autophagy in Pancreatic Cancer. Autophagy 17, 3275-3296. doi:10.1080/15548627.2020.1847462

Li, J., Liu, J., Xu, Y., Wu, R., Chen, X., Song, X., et al. (2021a). Tumor Heterogeneity in Autophagy-dependent Ferroptosis. Autophagy 17, 3361-3374. doi:10.1080/ 15548627.2021.1872241

Liu, J., Song, X., Kuang, F., Zhang, Q., Xie, Y., Kang, R., et al. (2021). NUPR1 Is a Critical Repressor of Ferroptosis. Nat. Commun. 12, 647. doi:10.1038/s41467021-20904-2

Liu, Y., Wang, Y., Liu, J., Kang, R., and Tang, D. (2021). Interplay between MTOR and GPX4 Signaling Modulates Autophagy-dependent Ferroptotic Cancer Cell Death. Cancer Gene Ther. 28, 55-63. doi:10.1038/s41417-020-0182-y

Ma, X., Xiao, L., Liu, L., Ye, L., Su, P., Bi, E., et al. (2021). CD36-mediated Ferroptosis Dampens Intratumoral CD8+ T Cell Effector Function and Impairs Their Antitumor Ability. Cell Metab 33, 1001-e5. doi:10.1016/ j.cmet.2021.02.015

Nagakannan, P., Islam, M. I., Conrad, M., and Eftekharpour, E. (2021). Cathepsin B Is an Executioner of Ferroptosis. Biochim. Biophys. Acta Mol. Cel Res 1868, 118928. doi:10.1016/j.bbamcr.2020.118928

Ran, Q., Gu, M., Van Remmen, H., Strong, R., Roberts, J. L., and Richardson, A. (2006). Glutathione Peroxidase 4 Protects Cortical Neurons from Oxidative Injury and Amyloid Toxicity. J. Neurosci. Res. 84, 202-208. doi:10.1002/ jnr.20868

Qu, C., Dai, E., Lai, T., Cao, G., Liu, J., Kang, R., et al. (2021). Itaconic Acid Induces Ferroptosis by Activating Ferritinophagy. Biochem. Biophys. Res. Commun. 583, 56-62. doi:10.1016/j.bbrc.2021.10.054

Shi, Z., Zhang, L., Zheng, J., Sun, H., and Shao, C. (2021). Ferroptosis: Biochemistry and Biology in Cancers. Front. Oncol. 11, 579286. doi:10.3389/ fonc. 2021.579286

Siegel, R. L., Miller, K. D., Fuchs, H. E., and Jemal, A. (2021). Cancer Statistics, 2021. CA A. Cancer J. Clin. 71, 7-33. doi:10.3322/caac.21654

Song, X., Liu, J., Kuang, F., Chen, X., Zeh, H. J., 3rd, Kang, R., et al. (2021). PDK4 Dictates Metabolic Resistance to Ferroptosis by Suppressing Pyruvate Oxidation and Fatty Acid Synthesis. Cell Rep 34, 108767. doi:10.1016/ j.celrep.2021.108767

Song, X., Zhu, S., Chen, P., Hou, W., Wen, Q., Liu, J., et al. (2018). AMPK-mediated BECN1 Phosphorylation Promotes Ferroptosis by Directly Blocking System Xc- Activity. Curr. Biol. 28, 2388-e5. doi:10.1016/j.cub.2018.05.094

Stockwell, B. R., Friedmann Angeli, J. P., Bayir, H., Bush, A. I., Conrad, M., Dixon, S. J., et al. (2017). Ferroptosis: A Regulated Cell Death Nexus Linking Metabolism, Redox Biology, and Disease. Cell 171, 273-285. doi:10.1016/ j.cell.2017.09.021

Su, Y., Zhao, B., Zhou, L., Zhang, Z., Shen, Y., Lv, H., et al. (2020). Ferroptosis, a Novel Pharmacological Mechanism of Anti-cancer Drugs. Cancer Lett. 483, 127-136. doi:10.1016/j.canlet.2020.02.015

Tang, D., Chen, X., Kang, R., and Kroemer, G. (2021). Ferroptosis: Molecular Mechanisms and Health Implications. Cell Res. 31, 107-125. doi:10.1038/ s41422-020-00441-1

Tang, D., Kepp, O., and Kroemer, G. (2020b). Ferroptosis Becomes Immunogenic: Implications for Anticancer Treatments. Oncoimmunology 10, 1862949. doi:10.1080/2162402X.2020.1862949

Tang, D., and Kroemer, G. (2020a). Ferroptosis. Curr. Biol. 30, R1292-R1297. doi:10.1016/j.cub.2020.09.068

Vander Heiden, M. G., Cantley, L. C., and Thompson, C. B. (2009). Understanding the Warburg Effect: the Metabolic Requirements of Cell Proliferation. Science 324, 1029-1033. doi:10.1126/science.1160809

Viswanathan, V. S., Ryan, M. J., Dhruv, H. D., Gill, S., Eichhoff, O. M., SeashoreLudlow, B., et al. (2017). Dependency of a Therapy-Resistant State of Cancer Cells on a Lipid Peroxidase Pathway. Nature 547, 453-457. doi:10.1038/ nature 23007

Wang, K., Zhang, Z., Tsai, H.-i., Liu, Y., Gao, J., Wang, M., et al. (2020). Branched-chain Amino Acid Aminotransferase 2 Regulates Ferroptotic Cell 
Death in Cancer Cells. Cell Death Differ 28, 1222-1236. doi:10.1038/s41418020-00644-4

Wenzel, S. E., Tyurina, Y. Y., Zhao, J., St Croix, C. M., Dar, H. H., Mao, G., et al. (2017). PEBP1 Wardens Ferroptosis by Enabling Lipoxygenase Generation of Lipid Death Signals. Cell 171, 628-e26. doi:10.1016/ j.cell.2017.09.044

Xie, Y., Hou, W., Song, X., Yu, Y., Huang, J., Sun, X., et al. (2016). Ferroptosis: Process and Function. Cel Death Differ 23, 369-379. doi:10.1038/ cdd.2015.158

Yan, B., Ai, Y., Sun, Q., Ma, Y., Cao, Y., Wang, J., et al. (2021). Membrane Damage during Ferroptosis Is Caused by Oxidation of Phospholipids Catalyzed by the Oxidoreductases POR and CYB5R1. Mol. Cel 81, 355-369. doi:10.1016/ j.molcel.2020.11.024

Yang, W. S., Kim, K. J., Gaschler, M. M., Patel, M., Shchepinov, M. S., and Stockwell, B. R. (2016). Peroxidation of Polyunsaturated Fatty Acids by Lipoxygenases Drives Ferroptosis. Proc. Natl. Acad. Sci. U S A. 113, E4966-E4975. doi:10.1073/pnas.1603244113

Yang, W. S., SriRamaratnam, R., Welsch, M. E., Shimada, K., Skouta, R., Viswanathan, V. S., et al. (2014). Regulation of Ferroptotic Cancer Cell Death by GPX4. Cell 156, 317-331. doi:10.1016/j.cell.2013.12.010

Yuan, H., Li, X., Zhang, X., Kang, R., and Tang, D. (2016). Identification of ACSL4 as a Biomarker and Contributor of Ferroptosis. Biochem. Biophys. Res. Commun. 478, 1338-1343. doi:10.1016/j.bbrc.2016.08.124

Zhang, Y., Tan, H., Daniels, J. D., Zandkarimi, F., Liu, H., Brown, L. M., et al. (2019). Imidazole Ketone Erastin Induces Ferroptosis and Slows Tumor Growth in a Mouse Lymphoma Model. Cel Chem Biol 26, 623-e9. doi:10.1016/j.chembiol.2019.01.008
Zhu, S., Zhang, Q., Sun, X., Zeh, H. J., 3rd, Lotze, M. T., Kang, R., et al. (2017) HSPA5 Regulates Ferroptotic Cell Death in Cancer Cells. Cancer Res. 77, 2064-2077. doi:10.1158/0008-5472.CAN-16-1979

Zou, Y., Henry, W. S., Ricq, E. L., Graham, E. T., Phadnis, V. V., Maretich, P., et al. (2020). Plasticity of Ether Lipids Promotes Ferroptosis Susceptibility and Evasion. Nature 585, 603-608. doi:10.1038/s41586-020-2732-8

Zou, Y., Li, H., Graham, E. T., Deik, A. A., Eaton, J. K., Wang, W., et al. (2020). Cytochrome P450 Oxidoreductase Contributes to Phospholipid Peroxidation in Ferroptosis. Nat. Chem. Biol. 16, 302-309. doi:10.1038/s41589-020-0472-6

Conflict of Interest: The authors declare that the research was conducted in the absence of any commercial or financial relationships that could be construed as a potential conflict of interest.

Publisher's Note: All claims expressed in this article are solely those of the authors and do not necessarily represent those of their affiliated organizations, or those of the publisher, the editors and the reviewers. Any product that may be evaluated in this article, or claim that may be made by its manufacturer, is not guaranteed or endorsed by the publisher.

Copyright $\odot 2021$ Liu, Kang and Tang. This is an open-access article distributed under the terms of the Creative Commons Attribution License (CC BY). The use, distribution or reproduction in other forums is permitted, provided the original author(s) and the copyright owner(s) are credited and that the original publication in this journal is cited, in accordance with accepted academic practice. No use, distribution or reproduction is permitted which does not comply with these terms. 